\title{
PERFIL DE MORTALIDADE POR CÂNCER DE COLO DO ÚTERO NO PERÍODO DE 2005-2014
}

\section{MORTALITY PROFILE FOR CERVICAL CANCER IN THE PERIOD 2005-2014}

\section{PERFIL DE MORTALIDAD PARA EL CÁNCER CERVICAL EN EL PERIODO 2005-2014}

Rejane Ceolin ${ }^{1}$, Cíntia Nasi², Adriana Aparecida Paz ${ }^{3}$, Graciele Fernanda da Costa Linch ${ }^{3}$

\section{RESUMO}

Objetivo: Identificar o perfil de mortalidade por câncer de colo do útero no Brasil. Método: Estudo de série histórica, com utilização de dados secundários provenientes do banco de dados do Instituto Nacional do Câncer, disponíveis no site do Departamento de Estatística do Sistema Único de Saúde. Resultados: Observou-se um aumento do número de óbitos por câncer de colo do útero no país no período estudado; contudo, por meio da análise das taxas, percebe-se um declínio da mortalidade no país, no estado e no município. Foi encontrado maior número de mortalidade entre 50 e 59 anos de idade, no país e no estado. Com relação às taxas de mortalidade, estas tiveram destaque a partir dos 80 anos de idade nas três esferas políticas administrativas. Conclusão: Os achados sugerem a necessidade de esforços no aprimoramento e ampliação das estratégias preventivas que atendam às necessidades da população nos três âmbitos, no intuito de diminuir a incidência de mortalidade pelo câncer de colo do útero.

Descritores: Neoplasias do colo do útero; Mortalidade; Saúde da mulher.

\section{ABSTRACT}

Objective: To identify the mortality profile of cervical cancer in Brazil. Method: A historical series study, using secondary data from the National Cancer Institute database, available on the Unified Health System Department of Statistics website. Results: There was an increase in the number of cervical cancer deaths in the country in the period studied; however, through the rates analysis, a decline in mortality in the country, state and municipality was noticed. It was found a higher number of mortality between 50 and 59 years in the country and in the state. Regarding to the mortality rates, these were highlighted from the age of 80 onwards in the three administrative policy areas. Conclusion: The findings suggest the need for efforts to improve and expand preventive strategies that meet the population needs in all three areas, in order to reduce the incidence of cervical cancer mortality.

escriptors: Uterine cervical neoplasms; Mortality; Women's health.

\section{RESUMEN}

Objetivo: Identificar la mortalidad por cáncer de cuello uterino en Brasil. Método: Estudio de serie histórica, con utilización de datos secundarios de la base de datos del Instituto Nacional del Cáncer, disponible en el sitio web del Departamento de Estadística del Sistema de Salud. Resultados: Hubo un aumento en el número de muertes por cáncer de cuello uterino en el país durante el período de estudio; sin embargo, por el análisis de las tasas, se observa un descenso de la mortalidad en el país, el estado y el condado. Se encontró mayor número de mortalidad entre 50 y 59 años de edad en el país y el estado. Con respecto a las tasas de mortalidad, estas se han destacado a partir de los 80 años de edad en las tres esferas políticas administrativas. Conclusión: Los resultados sugieren la necesidad de esfuerzos en la mejora y ampliación de las estrategias de prevención que satisfagan las necesidades de la población en las tres áreas, con el fin de reducir la incidencia de la mortalidad por cáncer de cuello de útero. Descriptores: Neoplasias del cuello uterino; Mortalidad; Salud de la mujer.

${ }^{1}$ Graduada em Enfermagem. Mestre em Enfermagem pela Universidade Federal de Ciências da Saúde de Porto Alegre - UFCSPA. ${ }^{2}$ Graduada em Enfermagem. Doutora em Enfermagem pela Universidade Federal do Rio Grande do Sul. Professora Adjunta da Escola de Enfermagem na Universidade Federal do Rio Grande do Sul - UFRGS. ${ }^{3}$ Graduada em Enfermagem. Doutora em Enfermagem pela Universidade Federal do Rio Grande do Sul. Professora Adjunta na Universidade Federal de Ciências da Saúde de Porto Alegre - UFCSPA.

Como citar este artigo:

Ceolin R, Nasi C, Paz AA, et al. Perfil de Mortalidade Por Câncer de Colo do Útero no Período de 2005-2014. Revista de Enfermagem do Centro-Oeste Mineiro.2018;8:e1806. [Access__]; Available in:___ DOI: https://doi.org/10.19175/recom.v7i0.1806 


\section{INTRODUÇÃO}

O câncer de colo do útero representa importante problema de saúde pública. Foi estimado, para o ano de 2016, o surgimento de 16.340 casos novos dessa neoplasia no Brasil, com um risco de 15,85 casos a cada 100 mil mulheres e com perspectiva de ocupar o quarto lugar entre os tipos de câncer mais frequentes entre as mulheres no biênio 2016-2017, perdendo para os cânceres de pele não melanoma, de mama e intestino. Relacionado com condições socioeconômicas menos favoráveis, o câncer de colo do útero, em geral, tem início a partir dos 30 anos e aumenta rapidamente seu risco até atingir os 50 anos ou mais ${ }^{(1)}$.

Apesar de ter evolução lenta, esse tipo de câncer apresenta altas taxas de incidência e mortalidade na população feminina, mobilizando gestores e profissionais de saúde no desenvolvimento de ações voltadas à melhoria dos indicadores de saúde dessa população. Nesse sentido, o fortalecimento da Saúde da Mulher no Brasil deve se dar através da implementação de políticas públicas e do rastreamento do câncer de colo do útero de forma efetiva ${ }^{(2)}$.

A infecção pelo papilomavírus humano (HPV) constitui o principal fator de risco para o desenvolvimento do câncer de colo do útero e representa uma das doenças sexualmente transmissíveis mais comuns no mundo. As infecções persistentes pelo HPV podem levar ao desenvolvimento de lesões intraepiteliais progressivas. Se não detectadas e tratadas adequadamente e de forma oportuna, podem evoluir para a neoplasia ${ }^{(1)}$.

A prevenção através da realização do exame preventivo constitui a ação mais efetiva na detecção precoce do câncer de colo uterino, pois possibilita rastrear a doença em estágio inicial, proporcionando, à mulher, tratamento e cura em tempo oportuno. Além disso, o exame é oferecido pela atenção primária de saúde ${ }^{(3)}$. Contudo, o sucesso do rastreamento está relacionado com as condições de acesso aos serviços de saúde e ao diagnóstico precoce ${ }^{(4)}$.

De acordo com as orientações do Ministério da Saúde, o rastreamento deve ser realizado dos 25 aos 64 anos de idade, em mulheres que já tenham iniciado a atividade sexual, sendo que, após dois exames realizados com intervalo de um ano, havendo resultado negativo, se deve seguir realizando os próximos a cada três anos. Devem ser interrompidos quando, após 64 anos e sem história prévia de lesões pré-neoplásicas, tiver dois exames negativos consecutivos nos últimos cinco anos ${ }^{(5)}$.

Diante disso e considerando a alta incidência do câncer de colo do útero, surgiu o questionamento sobre o perfil de mortalidade por esse tipo de câncer no município de Três Passos-RS, no estado do Rio Grande do Sul e no Brasil. Por ser o município de Três Passos local de trabalho de uma das pesquisadoras que atua junto a uma Estratégia Saúde da Família (ESF), realizando o rastreamento do câncer de colo do útero por meio do exame citopatológico, surgiu a inquietação com relação à mortalidade por esse tipo de neoplasia.

Para responder ao questionamento, o estudo buscou identificar o perfil de mortalidade por câncer de colo do útero em Três Passos, no Rio Grande do Sul e no Brasil no período de 2005 a 2014. A compreensão da situação de mortalidade por essa neoplasia permitirá compreender o padrão de ocorrência e evolução em diferentes locais, bem como avaliar os programas de rastreamento.

\section{MÉTODOS}

Este constitui um estudo descritivo, retrospectivo de série histórica ${ }^{(6)}$. Foram utilizados dados secundários, provenientes do banco de dados do Instituto Nacional do Câncer (INCA), disponíveis no site do Departamento de Estatística do Sistema Único de Saúde (DATASUS), sobre a mortalidade por câncer de colo do útero.

A coleta de dados ocorreu em novembro de 2016, e o período selecionado foi de 2005 a 2014, sendo a série histórica de 10 anos (inclui o período de janeiro de 2005 a dezembro de $2014=10$ anos). As variáveis utilizadas foram: neoplasia maligna do colo do útero (Código Internacional de Doenças CID 10 C53), período, local (Brasil, RS, Três Passos), e faixa etária conforme o local de ocorrência.

De acordo com o último Censo, realizado em 2010, o município de Três Passos conta com uma população estimada de 24.640 habitantes, sendo 12.214 mulheres e, destas, 6.684 estão entre a população-alvo para o rastreamento do câncer de colo do útero, de 25 a 64 anos $^{(7)}$.

A análise dos dados foi por meio de estatística descritiva de frequência absoluta.

O estudo da mortalidade por análise da tendência temporal constitui fonte de informação de apoio à política de tomada de decisão e auxilia a avaliação do impacto de medidas preventivas sendo implementadas ${ }^{(4)}$.

Foram respeitados os aspectos éticos, previstos na Resolução n.o 466/2012, para o uso de 
dados secundários. Por ser o INCA e o DATASUS bases de dados secundários de domínio público, não foi necessária avaliação do Comitê de Ética.

\section{RESULTADOS E DISCUSSÃO}

Os dados são apresentados por meio de figuras para ilustrar a mortalidade por câncer de colo do útero entre as três esferas político-administrativas.
A Figura 1 apresenta a mortalidade proporcional por câncer de colo do útero em mulheres, no Brasi( ${ }^{(8)}$.

Observa-se crescimento de óbitos por câncer de colo do útero no Brasil entre os anos 2005 e 2014. Em 2005, foram registrados 4.506 óbitos. Já no ano de 2014, um total de 5.448 óbitos por câncer de colo do útero foram registrados no país.

Figura 1 - Mortalidade proporcional não ajustada por câncer de colo do útero, mulheres, Brasil, entre 2005 e 2014.

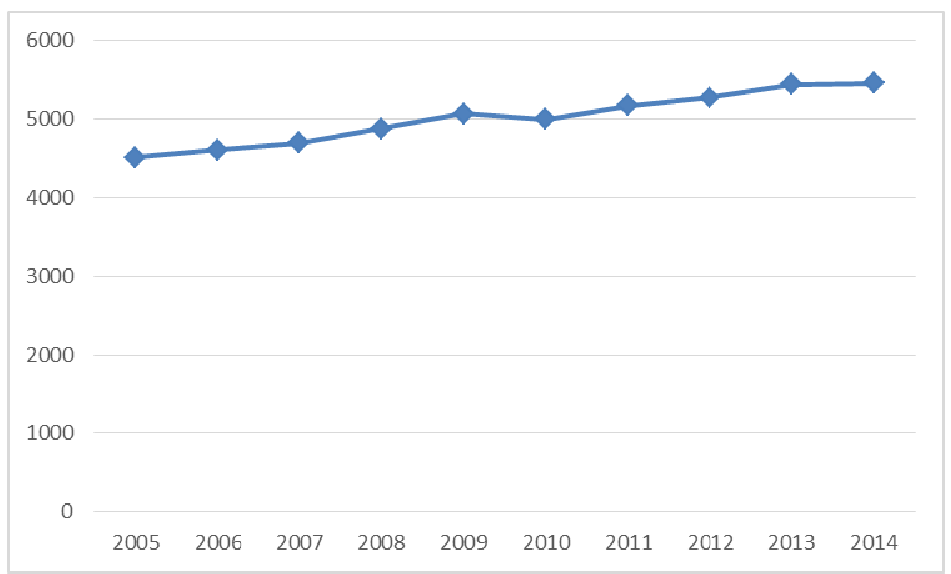

Fonte: INCA/Datasus/MS, 2016.

Ao observar um aumento do número de óbitos por câncer de colo do útero no país, compreende-se que esse fato pode ter relação com o processo de transição epidemiológica em andamento, com o aumento das doenças crônicas, nelas incluídas o câncer, e, consequentemente, o aumento direto da mortalidade por esse tipo de neoplasia ${ }^{(9,10)}$. Essa transição epidemiológica é caracterizada pela diminuição dos registros de doenças transmissíveis e pelo aumento das doenças crônicas não transmissíveis. Somado a isso, as pessoas estão vivendo mais. Essa longevidade encontra relação com o surgimento das doenças crônicas, como o câncer.

A Figura 2 apresenta a mortalidade proporcional por câncer de colo do útero em mulheres, na região Sul(8).

Figura 2 - Mortalidade proporcional não ajustada por câncer de colo do útero, mulheres, região Sul, entre 2005 e 2014.

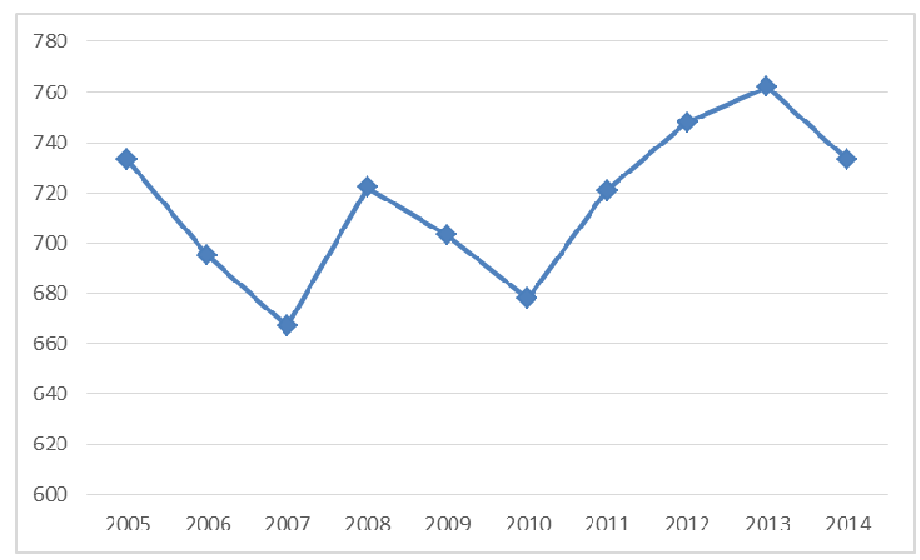

Fonte: INCA/Datasus/MS, 2016.

$\mathrm{Na}$ região Sul, no ano de 2005, foram registrados 733 óbitos por câncer de colo do útero. Observa-se uma queda dos óbitos para 667 no ano de 2007. Posteriormente, chama a atenção um considerável aumento em 2013, apresentando 762 óbitos, com pequeno declínio em 2014 e retorno a 
733 óbitos, o mesmo número encontrado no ano de 2005. Dessa maneira, percebe-se forte oscilação entre os anos pesquisados, ao contrário do parâmetro do país, cujo crescimento segue uma tendência de aumento linear. Tal diferença entre os anos estudados pode ocorrer devido à subnotificação de casos de mortalidade.

Segundo a análise das projeções de mortalidade no Brasil até 2030, haverá uma redução das taxas, com destaque para a região Sul. Essa redução encontra explicação na diminuição dos riscos para o desenvolvimento do câncer de colo do útero. Entretanto, as regiões Norte e Nordeste apresentam as maiores taxas, o que indica que a mortalidade está relacionada com as regiões mais pobres do Brasil. Devido às diferenças regionais, considera-se desafiador o controle da doença no país ${ }^{(11)}$.

De acordo com as taxas de mortalidade das cinco localizações primárias mais frequentes encontradas no INCA ${ }^{(8)}$ entre os anos 2005 e 2014, o câncer de colo do útero ocupa o terceiro lugar entre os cânceres que mais causam mortalidade no Brasil, ficando atrás somente dos cânceres de mama e de brônquios e pulmões.

Com o ajuste das taxas para 100.000 mulheres, em 2005, foi encontrado um percentual de 5,29 óbitos para o câncer de colo do útero. Já em 2014, esse percentual foi de 4,88. Dessa maneira, por meio da análise das taxas, percebe-se um declínio da mortalidade no país. Esse dado difere daquele apresentado anteriormente sobre a mortalidade proporcional por câncer de colo do útero em mulheres, no Brasil, no mesmo período de tempo.

No Rio Grande do Sul( ${ }^{(8)}$, a mortalidade por câncer de colo do útero ocupa o quinto lugar, ficando atrás dos de mama, brônquios e pulmões, cólon, e pâncreas. Da mesma forma que no país, o número de óbitos diminui nesse período de tempo, com o registro de 5,4 óbitos para 100.000 mulheres em 2005, e 4,08 em 2014.

Já no município de Três Passos ${ }^{(8)}$, pode-se afirmar que o câncer de colo do útero não ocupa lugar significativo, pois não está entre as cinco neoplasias mais frequentes, diferentemente da situação do país e do estado do Rio Grande do Sul.

Essas tendências refletem as medidas de rastreamento através do exame Papanicolau e de controle da transmissão pelo HPV adotadas principalmente após 2004 $4^{(4)}$. Além disso, a estruturação das ESFs no município possibilitou fornecer ampla cobertura para a população.

A tendência decrescente das taxas de mortalidade por câncer de colo do útero no país, no Rio Grande do Sul e em Três Passos é semelhante à observada em um estudo de série histórica realizado em um município de Minas Gerais, onde foi encontrada queda da mortalidade por essa neoplasia. Segundo uma análise de tendências, o resultado sugere um processo de transição epidemiológica em andamento, com persistência de taxas elevadas do câncer de colo do útero( ${ }^{(9)}$.

As taxas de mortalidade por câncer de colo do útero apresentam flutuações significativas ao longo do tempo, influenciadas pelas condições de acesso aos serviços de saúde para a prevenção primária contra o HPV, bem como a prevenção secundária para o rastreamento e o diagnóstico precoce. Diante disso, torna-se complexo detectar se há aumento ou redução progressiva nas taxas de mortalidade por câncer no Brasil e estimar seu valor através da análise em série temporal ${ }^{(4)}$.

$\mathrm{Na}$ Figura 3, apresentam-se as taxas de mortalidade por câncer de colo do útero, brutas e ajustadas por idade para 100.000 mulheres, no $\mathrm{Brasi}^{(8)}$.

Figura 3 - Taxas de mortalidade por câncer de colo do útero, brutas e ajustadas por idade, pelas populações mundial e brasileira de 2010, por 100.000 mulheres, Brasil, entre 2005 e 2014.

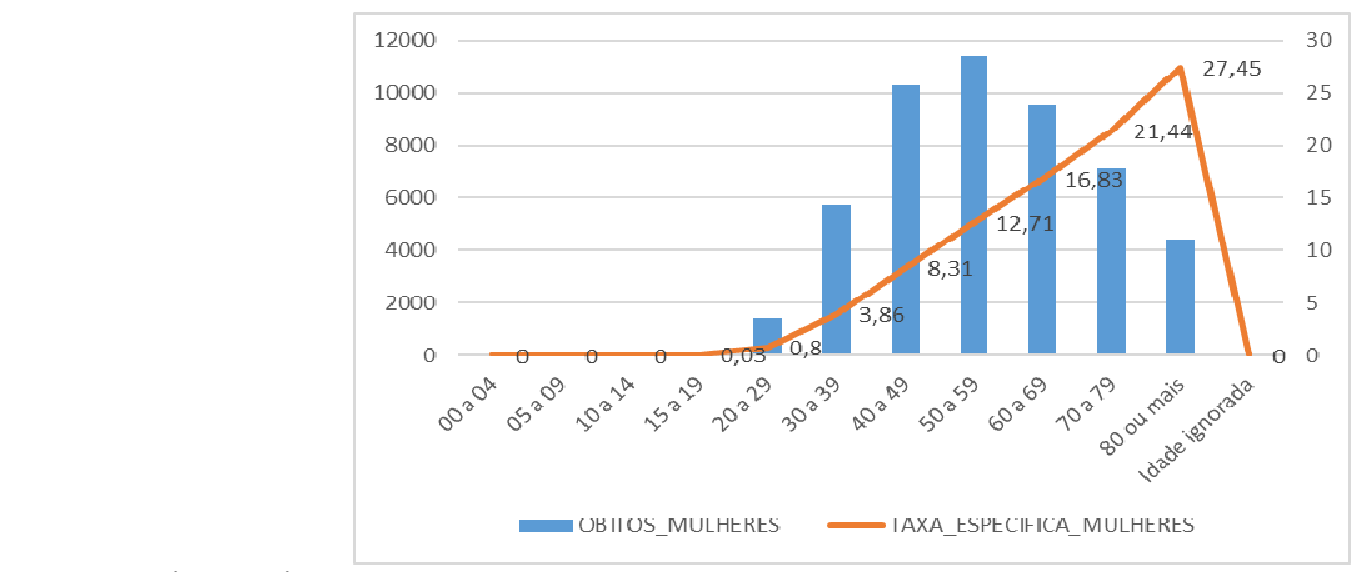

Fonte: INCA/Datasus/MS, 2016. 
No Brasil, chama-se atenção à diferença entre o número de óbitos e taxas de óbitos por idade. Observa-se que, entre 50 e 59 anos de idade, foi maior o número de registros de mortalidade, com 11.423 óbitos, o que equivale à taxa de 12,71 . Aos 80 anos ou mais, 4.456 óbitos foram identificados, sendo a taxa de 27,45 . Diante disso, considera-se que tal diferença pode ocorrer por a população feminina, a partir dos 80 anos, ser menor. Sendo assim, essa diferença encontrada torna-se significativa para a avaliação, - que leva ao entendimento de que mais mulheres morrem pela neoplasia, no país, com 80 anos de idade ou mais.

A Figura 4 mostra as taxas de mortalidade por câncer de colo do útero, brutas e ajustadas por idade, por 100.000 mulheres, no Rio Grande do Sul( ${ }^{(8)}$.

Figura 4 - Taxas de mortalidade por câncer de colo do útero, brutas e ajustadas por idade, pelas populações mundial e brasileira de 2010, por 100.000 mulheres, Rio Grande do Sul, entre 2005 e 2014.

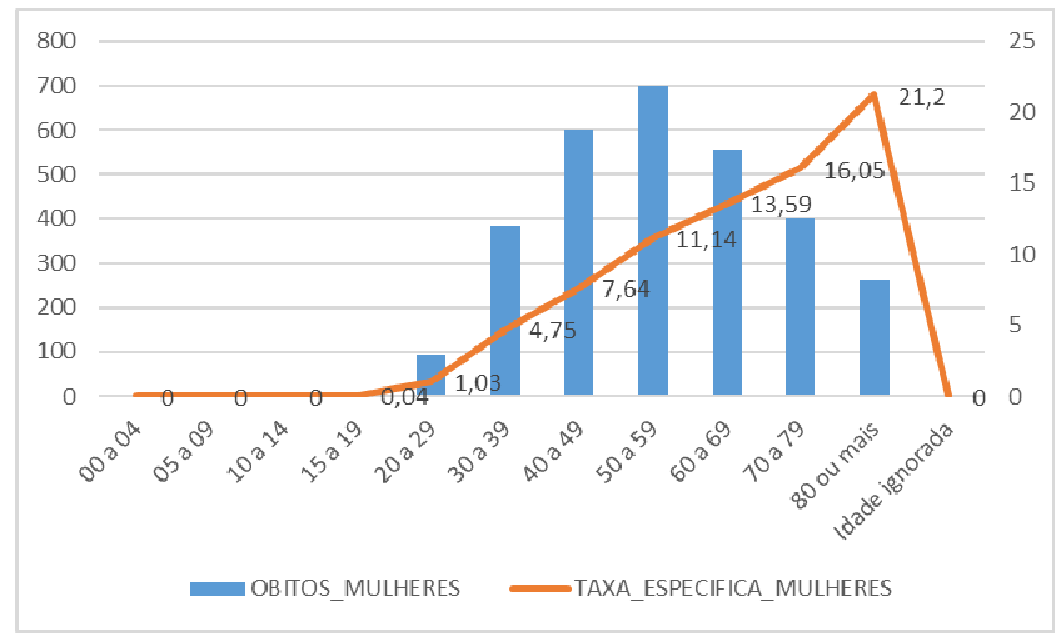

Fonte: INCA/Datasus/MS, 2016.

No Rio Grande do Sul, observam-se proporções parecidas com as do país com relação ao número de óbitos e às taxas de mortalidade. 0 maior número de óbitos foi entre 50 e 59 anos, com um registro de 699 óbitos. Já com 80 anos ou mais, 261 óbitos foram identificados.
A Figura 5 apresenta as taxas de mortalidade por câncer de colo do útero, brutas e ajustadas por idade, por 100.000 mulheres, no município de Três Passos ${ }^{(8)}$.

Figura 5 - Taxas de mortalidade por câncer de colo do útero, brutas e ajustadas por idade, pelas populações mundial e brasileira de 2010, por 100.000 mulheres, Três Passos-RS, entre 2005 e 2014.

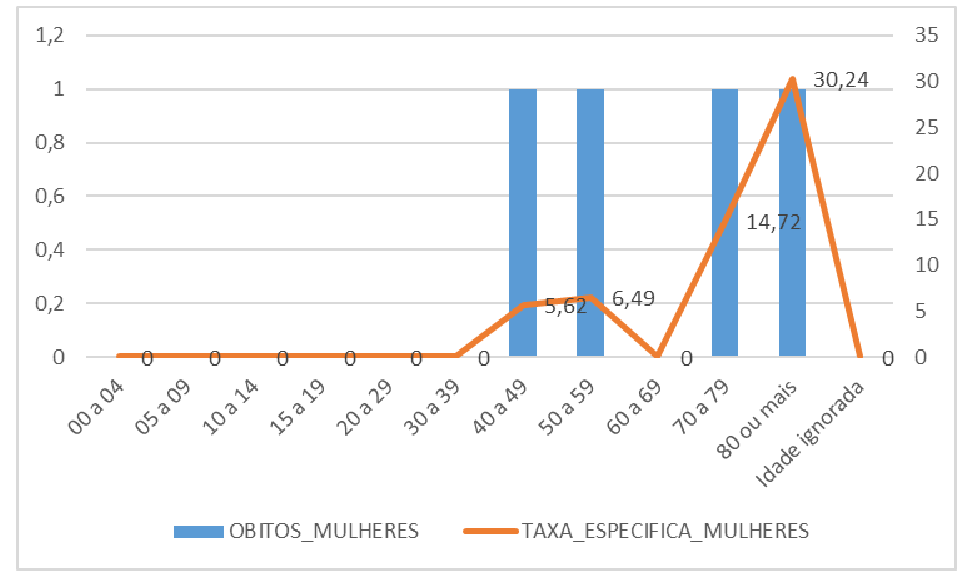

Fonte: INCA/Datasus/MS, 2016. 
No município de Três Passos, foram registrados quatro óbitos no mesmo período de estudo e, da mesma forma que no país e no estado, observa-se maior taxa de mortalidade para os 80 anos ou mais de idade. Entretanto, chama a atenção o reduzido número de óbitos registrados no município durante a série de 10 anos. Tal diferença pode encontrar relação com a subnotificação de casos de mortalidade no município no período estudado. Também pode encontrar relação com a necessidade de tratamento e hospitalização em outros municípios que sejam referência para os casos de câncer; como o registro de mortalidade é no local de ocorrência, diminui o registro no de residência.

A idade representa um fator de risco para a ocorrência do câncer devido à relação com o processo biológico de envelhecimento ${ }^{(4)}$. Dessa maneira, a análise da mortalidade por grupos etários apresenta associação com o envelhecimento ${ }^{(9)}$. Em um estudo, verificou-se um aumento na taxa de mortalidade por câncer de colo do útero a partir da faixa etária dos 40 aos 49 anos. Já dos 60 aos 69 e a partir dos 70 anos, concentraram-se $46,4 \%$ dos óbitos no período de 1999 a $2006^{(12)}$. Sendo assim, pode-se considerar que as mulheres idosas apresentam maior taxa de mortalidade por câncer de colo do útero.

Quando as taxas de mortalidade específicas por idade são estudadas durante determinado período de tempo, refletem alguns efeitos relacionados com $\mathrm{o}$ ano da morte e do nascimento e caracterizam as mudanças de mortalidade ao longo do tempo ${ }^{(4)}$. Desse modo, nota-se também a influência das transições demográficas sobre as taxas de mortalidade.

Da análise da mortalidade por câncer de colo do útero, alguns aspectos merecem destaque, decorrentes do processo de implementação do programa voltado à Saúde da Mulher no decorrer dos anos. Merece destaque a Portaria n.o 874/13, em que foi publicada a Política Nacional para a Prevenção e Controle do Câncer na Rede de Atenção à Saúde das pessoas com doenças crônicas no âmbito do SUS, trazendo diretrizes relacionadas à prevenção do câncer ${ }^{(13)}$.

Dentro dessa perspectiva, cabe destacar que, no município de Três Passos, a implantação dos serviços e programas voltados à saúde da mulher e ao combate ao câncer seguiu a cronologia de execução das políticas em nível nacional.
Um estudo transversal realizado em Campinas, no Brasil, com 2.220.298 exames citopatológicos, mostrou que o exame Papanicolau é efetivo para prevenir as lesões intraepiteliais escamosas citológicas de alto grau, carcinoma de células escamosas, adenocarcinoma in situ e adenocarcinoma invasivo quando realizado com intervalos menores de cinco anos ${ }^{(14)}$.

Todavia, apesar da efetividade do exame citopatológico, bem como previsão de redução nas taxas de mortalidade, estas ainda se mostram elevadas. No estado do Rio Grande do Norte, as projeções indicaram redução nas taxas de mortalidade de 5,95 por 100 mil mulheres (20062010) para 3,67 (2026-2030). Contudo, está previsto aumento de $22 \%$ no número absoluto de óbitos. Mesmo com o alcance de melhores indicadores de mortalidade, esse resultado permanece menor do que o esperado(10).

Nesse âmbito, problemas relacionados com - acesso aos serviços de saúde impedem o rastreamento e o diagnóstico precoce, bem como inviabilizam às mulheres a oportunidade de receber o tratamento em tempo oportuno. Essa dificuldade acomete principalmente mulheres com menores condições socioeconômicas e que dependem exclusivamente dos serviços públicos de saúde ${ }^{(9)}$. Diante disso, a busca pela universalidade de acesso aos serviços de saúde e do rastreamento precoce de forma efetiva constituem prioridades para melhorar os índices de mortalidade por câncer de colo do útero.

\section{CONCLUSÃO}

Com a realização deste estudo, foi possível identificar uma redução nas taxas de mortalidade por câncer de colo do útero no Brasil, no estado do Rio Grande do Sul e no município de Três Passos. Isso se deve às medidas adotadas no rastreamento precoce do câncer. Foi identificada também maior taxa de mortalidade a partir dos 80 anos de idade, a qual pode ter relação com os aspectos envolvendo as transições epidemiológicas e demográficas no país.

O perfil de mortalidade por câncer de colo do útero identificado neste estudo foi baseado nos dados informados pelo INCA. Sendo assim, podem apresentar limitações em decorrência da qualidade da informação gerada. Todavia, as medidas de prevenção e controle, realizadas nos serviços de saúde, possibilitam reduzir a mortalidade por essa neoplasia. 
Recomenda-se a realização de novos estudos para a análise da mortalidade em municípios de pequeno porte. Identificou-se que a maioria dos estudos sobre mortalidade por câncer de colo do útero se concentra em capitais ou estados da federação, sendo pouco conhecido o perfil de mortalidade em municípios pequenos.

Os achados sugerem a necessidade de esforços no aprimoramento e na ampliação das estratégias preventivas no âmbito das políticas públicas de saúde, tendo em vista a cobertura de um maior número de mulheres nas ações de promoção da saúde. Desse modo, o fortalecimento do programa de controle do câncer de colo do útero no país, bem como o acesso aos serviços de saúde, são considerados prioridades para que a redução na mortalidade identificada não estacione ao longo do tempo.

Acredita-se que este estudo poderá viabilizar o desenvolvimento de ações em saúde pública que atendam às necessidades da população nos três âmbitos, no intuito de diminuir a incidência de mortalidade pelo câncer de colo do útero.

\section{REFERÊNCIAS}

1. Ministério da Saúde (BR). Instituto Nacional de Câncer José Alencar Gomes da Silva (Inca). Estimativa 2016: incidência de câncer no Brasil. Rio de Janeiro: Instituto Nacional de Câncer José Alencar Gomes da Silva; 2015[citado em 18 nov 2016]. Disponível em: http://www.inca.gov.br/estimativa/2016/estimativ a-2016-v11.pdf

2. Silva DSM, Silva AMN, Brito LMO, Gomes SRL, Nascimento, Chein MBC. Rastreamento do câncer do colo do útero no Estado do Maranhão, Brasil. Cienc Saúde Coletiva. 2014;19(4):1163-70. https://doi.org/10.1590/1413-

\subsection{3}

3. Viana MRP, Moura MEB, Nunes BMVT, Monteiro CFS, Lago EC. Formação do enfermeiro para a prevenção do câncer de colo uterino. Rev Enferm UERJ. 2013;21(5):624-30. https://doi.org/10.12957/reuerj.2013.10038

4. Guimarães RM, Muzi CD, Ayres ARG, Ribeiro MS, Chagas CC, Oliveira JSC. Aplicação de três técnicas para avaliação de tendências de mortalidade por câncer do colo do útero em série temporal no Brasil, 1980-2009. Rev Bras Cancerol. 2012 [citado em 8 dez 2016];58(3):359-67. Disponível

em:

http://www1.inca.gov.br/rbc/n 58/v03/pdf/05 ar tigo aplicacao tres tecnicas avaliacao tendenci a mortalidade cancer colo utero serie tempor

al brasil 1980 2009.pdf

5. Ministério da Saúde (BR). Protocolos da Atenção Básica: saúde das mulheres. Brasília, DF: Ministério da Saúde, 2016 [citado em 8 dez 2016]. Disponível

em:

http://189.28.128.100/dab/docs/portaldab/public acoes/protocolo saude mulher.pdf

6. Rouquayrol MZ. Epidemiologia \& saúde. 4a ed. São Paulo: Medsi; 2004.

7. Instituto Brasileiro de Geografia e Estatística (IBGE). Censo demográfico: cidades. Rio de Janeiro: Instituto Brasileiro de Geografia e Estatística; 2010 [citado em 8 dez 2016]. Disponível em: http://www.cidades.ibge.gov.br/xtras/perfil.php?! ang $=\&$ codmun $=432190$

8. Ministério da Saúde (BR). Departamento de Informática do Sistema Único de Saúde. Estatísticas vitais: câncer. Brasília, DF: Ministério da Saúde; 2016[citado em 18 nov 2016]. Disponível em: http://www2.datasus.gov.br/DATASUS/index.php ?area $=0205 \& i d=19465$

9. Rodrigues $A D$, Bustamante-Teixeira MT. Mortalidade por câncer de mama e câncer de colo do útero em município de porte médio da Região Sudeste do Brasil, 1980-2006. Cad Saúde Pública.

2011;27(2):241-8.

https://doi.org/10.1590/S0102-

311X2011000200005

10. Sousa AMV, Teixeira CCA, Medeiros SS, Nunes SJC, Salvador PTCO, Barros RMB et al. Mortalidade por câncer do colo do útero no estado do Rio Grande do Norte, no período de 1996 a 2010: tendência temporal e projeções até 2030. Epidemiol Serv Saúde. 2016;25(2):311-22. https://doi.org/10.5123/s1679-49742016000200010 11. Barbosa IR, Souza DLB, Bernal MM, Costa ICC. Desigualdades regionais na mortalidade por câncer de colo de útero no Brasil: tendências e projeções até o ano 2030. Cienc Saúde Coletiva. 2016;21(1):253-62. https://doi.org/10.1590/141381232015211.03662015

12. Meira KC, Gama SGN, Silva CMFP. Perfil de mortalidade por câncer do colo do útero no município do Rio de Janeiro no período 19992006. Rev Bras Cancerol. 2011[citado em 29 nov 2016];57(1):7-14. Disponível em: http://www.inca.gov.br/rbc/n 57/v01/pdf/03 arti go perfil mortalidade cancer utero rio de jane iro 1999.pdf

13. Ministério da Saúde (BR). Instituto Nacional de Câncer José Alencar Gomes da Silva (Inca). 
Coordenação de Prevenção e Vigilância. Manual de gestão da qualidade para laboratório de citopatologia. 2a ed rev ampl. Rio de Janeiro: Instituto Nacional de Câncer José Alencar Gomes da Silva; 2016[citado em 8 dez 2016]. Disponível em:

http://www1.inca.gov.br/inca/Arquivos/livro co mpleto manual citopatologia.pdf

14. Vale, DB, Braganca JF, Morais SS, Zeferino LC. Protection against squamous cell carcinoma and cervical adenocarcinoma afforded by cervical cytology screening: a cross-sectional study. Int J Gynecol Cancer. 2014;24(2):321-8. https://doi.org/10.1097/IGC.0000000000000057

Nota: Este estudo é um produto do Curso de Mestrado Profissional em Enfermagem da Universidade Federal de Ciências da Saúde de Porto Alegre (UFCSPA), desenvolvido na disciplina de Sistemas de Informação em Saúde Pública.

Recebido em: 20/02/2017

Aprovado em: 28/11/2017

Endereço de correspondência:

Rejane Ceolin

Avenida dos Estados, № 1620 - Bela Vista CEP: 98600-000 - Três Passos/FS - Brasil

E- mail: rejane.ceolin@hotmail.com 